\title{
Biomedical Fibers and Nanofibers
}

Xiangyang Shi ${ }^{1}$

๑) Donghua University, Shanghai, China 2020

Fiber materials represent a class of important one-dimensional materials with unique properties for a range of applications in the fields of energy storage, catalysis, sensing, cleaner production, tissue engineering or other bio-related applications, besides their traditional applications in textile industry. In particular, nanofibers with a diameter less than $1000 \mathrm{~nm}$ display a relatively high surface area to volume ratio, abundant surface modification sites, desired orientation or aggregation, as well as tunable surface topographic features. These characteristics render fibers and nanofibers with a range of biomedical applications including but not limited to drug delivery, tissue engineering, and biosensing applications.

To showcase recent advances of fibers or nanofibers in biomedical applications, Advanced Fiber Materials organized this special issue on the topic of "Biomedical fibers and nanofibers". This issue collects together four contributions (one review article and three research papers) from active experts in the related fields and highlights the development of fibers or nanofibers for biosensing, tissue engineering, and drug delivery applications.

As a global view, X. Li, B. Ding and coworkers report recent advances on the use and design of electrospun nanofibrous materials for wound healing, emphasizing on the efforts for controlling fiber alignment and morphology, constructing 3D scaffolds, developing waterproof-breathable membrane, governing drug delivery performance, and regulating stem cell behavior. G. R. Williams, M. Orlu and colleagues designed coaxial core-shell electrospun fibers co-loaded with levodopa and carbidopa for potential treatment of Parkinson's disease. The design is beneficial to reduce the required dosing frequency. $Z$. Wang and R. Pei et al. developed a small peptide-modified multiscale $\mathrm{TiO}_{2}$ nanofibers generated through electrospinning for the selective enrichment and isolation of circulating tumor cells, which is proven to be a simple, inexpensive, and reliable method for potential diagnosis of early-stage cancer, prognosis, and monitoring therapeutic response. Lastly, X. Dou, C. Feng and coworkers report a chiral supramolecular hydrogel formed through the co-assembly of a two-component fibrous solution and sucrose for visual recognition of simple sugars, and the developed chiral co-assembly strategy for molecular recognition may be potentially used in medical diagnostics and cell imaging.

I would like to acknowledge the renowned authors in the field to contribute to this special issue by submitting their state of art reviews and high quality research papers as well as the reviewers for their vigorous and timely comments to ensure the quality of the publications. I would also sincerely thank editor-in-chief Prof. Meifang Zhu and the editorial team of Advanced Fiber Materials as well as my colleague Dr. Xueyan Cao to organize and publish such a wonderful special issue.
Xiangyang Shi

xshi@dhu.edu.cn

1 College of Chemistry, Chemical Engineering, and Biotechnology, Donghua University, Shanghai, China 